\title{
STABLE AND REAL-ZERO POLYNOMIALS IN TWO VARIABLES
}

\author{
ANATOLII GRINSHPAN, DMITRY S. KALIUZHNYI-VERBOVETSKYI, VICTOR VINNIKOV, \\ AND HUGO J. WOERDEMAN
}

\begin{abstract}
For every bivariate polynomial $p\left(z_{1}, z_{2}\right)$ of bidegree $\left(n_{1}, n_{2}\right)$, with $p(0,0)=1$, which has no zeros in the open unit bidisk, we construct a determinantal representation of the form

$$
p\left(z_{1}, z_{2}\right)=\operatorname{det}(I-K Z)
$$

where $Z$ is an $\left(n_{1}+n_{2}\right) \times\left(n_{1}+n_{2}\right)$ diagonal matrix with coordinate variables $z_{1}, z_{2}$ on the diagonal and $K$ is a contraction. We show that $K$ may be chosen to be unitary if and only if $p$ is a (unimodular) constant multiple of its reverse.

Furthermore, for every bivariate real-zero polynomial $p\left(x_{1}, x_{2}\right)$, with $p(0,0)=1$, we provide a construction to build a representation of the form

$$
p\left(x_{1}, x_{2}\right)=\operatorname{det}\left(I+x_{1} A_{1}+x_{2} A_{2}\right),
$$

where $A_{1}$ and $A_{2}$ are Hermitian matrices of size equal to the degree of $p$.

A key component of both constructions is a stable factorization of a positive semidefinite matrix-valued polynomial in one variable, either on the circle (trigonometric polynomial) or on the real line (algebraic polynomial).
\end{abstract}

\section{INTRODUCTION}

Stability of multivariate polynomials is an important concept arising in a variety of disciplines, such as Analysis, Electrical Engineering, and Control Theory [11, 58, 9, 37, 12, 23]. In this paper, we discuss two-variable polynomial stability with respect to the open unit bidisk

$$
\mathbb{D}^{2}=\left\{\left(z_{1}, z_{2}\right) \in \mathbb{C}^{2}:\left|z_{1}\right|<1,\left|z_{2}\right|<1\right\} .
$$

A bivariate polynomial will be called semi-stable if it has no zeros in $\mathbb{D}^{2}$, and stable if it has no zeros in the closure $\overline{\mathbb{D}}^{2}$. The bidegree of $p \in \mathbb{C}\left[z_{1}, z_{2}\right]$ is the pair $\operatorname{deg} p=\left(\operatorname{deg}_{1} p, \operatorname{deg}_{2} p\right)$ of its partial degrees in each variable. The reverse of $p$ is defined as $\overleftarrow{p}(z)=z^{\operatorname{deg} p} \bar{p}(1 / z)$, where for $z=\left(z_{1}, z_{2}\right)$ and $n=\left(n_{1}, n_{2}\right)$ we set $z^{n}=z_{1}^{n_{1}} z_{2}^{n_{2}}, \bar{p}(z):=\overline{p(\bar{z})}, \bar{z}=\left(\bar{z}_{1}, \bar{z}_{2}\right)$, and $1 / z=\left(1 / z_{1}, 1 / z_{2}\right)$. A polynomial is self-reversive if it agrees with its reverse 11 A semi-stable polynomial $p$ is scattering Schur [9] if $p$ and $\overleftarrow{p}$ are coprime, i.e., have no common factors.

For every semi-stable $p \in \mathbb{C}\left[z_{1}, z_{2}\right]$, with $p(0,0)=1$, we construct a representation

$$
p\left(z_{1}, z_{2}\right)=\operatorname{det}\left(I_{|n|}-K Z_{n}\right),
$$

1991 Mathematics Subject Classification. 15A15; 47A13, 13P15, 90C25.

Key words and phrases. Determinantal representation; multivariable polynomial; (semi-)stable polynomial; stability radius; self-reversive polynomial; real-zero polynomial; Lax conjecture.

AG, DK-V, HW were partially supported by NSF grant DMS-0901628. DK-V and VV were partially supported by BSF grant 2010432.

${ }^{1}$ The terminology adopted here is different from that in some other sources. E.g., one can find in the literature "stable" and "strictly stable" corresponding to our "semi-stable" and "stable", "dual" or "inverse" corresponding to our "reverse", and "unimodular" or "self-inversive" corresponding to our "self-reversive". 
with $n=\operatorname{deg} p$ and $K$ a contraction, where $|n|=n_{1}+n_{2}$ and $Z_{n}=z_{1} I_{n_{1}} \oplus z_{2} I_{n_{2}}$; see Theorem 2.1. Although we follow a slightly different path to achieve this result, we are essentially in the trail of Kummert [36, 35, 37], who established (1.1) in the case of scattering Schur polynomials [37, Theorem 1]. Note that, given a contractive $K$, every polynomial defined by (1.1) is semi-stable, so one gains practical means of designing semi-stable bivariate polynomials.

As an application of Theorem 2.1, we also establish in Theorem 3.2 a representation (1.1) for semi-stable self-reversive polynomials, with $K$ a unitary matrix; notice that semi-stable self-reversive polynomials are never scattering Schur. This representation was previously established directly in [18, Section 10] in a somewhat different setting.

In the one-variable case, the situation is transparent: every $p \in \mathbb{C}[z]$, with $p(0)=1$, can be written in the form

$$
p(z)=\left(1-a_{1} z\right) \cdots\left(1-a_{n} z\right)=\operatorname{det}\left(I_{n}-\left[\begin{array}{lll}
a_{1} & & \\
& \ddots & \\
& & a_{n}
\end{array}\right]\left[\begin{array}{lll}
z & & \\
& \ddots & \\
& & z
\end{array}\right]\right),
$$

where $1 / a_{i}$ are the zeros of $p$ counting multiplicities. Thus $p$ admits a representation (1.1), with $K=\operatorname{diag}\left[a_{1}, \ldots, a_{n}\right]$. This representation is minimal in size, $n=\operatorname{deg} p$, and in norm, $\|K\|=\max _{1 \leq i \leq d}\left|a_{i}\right|$. Observe that $p$ is semi-stable (respectively, stable) if and only if $K=\operatorname{diag}\left[a_{1}, \ldots, a_{n}\right]$ is contractive (respectively, strictly contractive); in particular, all zeros of $p$ are on the unit circle if and only if (1.1) holds with $K$ a diagonal unitary. We also note that $K$ can be chosen to have all, with perhaps one exception, singular values equal to 1. By a result of A. Horn [28], this choice is realized by an upper-triangular $K$ with eigenvalues $a_{1}, \ldots, a_{n}$ and singular values equal to $1, \ldots, 1,\left|\prod_{i=1}^{n} a_{i}\right|$; see also [29, Theorem 3.6.6].

Our main result, Theorem 2.1, shows that in the two-variable case we are as well able to find a representation (1.1) that is minimal both in the size and in norm of $K$. In particular, this means that for a two-variable semi-stable polynomial $p$ with $p(0,0)=1$, we can find a representation (1.1) with $n=\operatorname{deg} p$ and $K$ a contraction.

In three or more variables, such a result does not hold; indeed, it follows from [22, Example 5.1] that for $5 / 6<r<1$ the stable polynomial

$$
q\left(z_{1}, z_{2}, z_{3}\right)=1+\frac{r}{5} z_{1} z_{2} z_{3}\left(z_{1}^{2} z_{2}^{2}+z_{2}^{2} z_{3}^{2}+z_{3}^{2} z_{1}^{2}-2 z_{1} z_{2} z_{3}^{2}-2 z_{1} z_{2}^{2} z_{3}-2 z_{1}^{2} z_{2} z_{3}\right),
$$

does not have a representation (1.1) with $K$ a contractive $9 \times 9$ matrix, even though its degree is $(3,3,3)$. In general, the problem of finding a representation (1.1) with some (not necessarily contractive) matrix $K$ and $n=\operatorname{deg} p$ for a multivariable polynomial $p$, is overdetermined (see [22]). It is also unknown whether a semi-stable polynomial $p$ in more than two variables admits a representation of the form (1.1) with a contractive matrix $K$ of any size (see [22] for a discussion). An alternative certificate for stability in any number of variables is given in [59]. The general problem of constructing linear determinantal representations of a polynomial is a well known classical problem in algebraic geometry, see [32] and the references therein.

The analog of semi-stable self-reversive polynomials for the real line (as opposed to the unit circle in the complex plane) are real-zero polynomials (or - upon homegenization - homogeneous hyperbolic polynomials first introduced by Gårding [16, 17]). These polynomials and their determinantal representations were actively studied in recent years in relation to semidefinite programming; we refer to [57] for a state of the art survey and further references. 
Using stable factorization of univariate matrix polynomials that are positive semidefinite on the real line, we construct in Section 4 a positive self-adjoint determinantal representation for two-variable real-zero polynomials, i.e., a determinantal representation of the form $p\left(x_{1}, x_{2}\right)=p(0,0) \operatorname{det}\left(I+x_{1} A_{1}+x_{2} A_{2}\right)$ where $A_{1}$ and $A_{2}$ are complex self-adjoint matrices of the size equal to the degree of $p$. This reproves the main result of [26], see also [25] (which amounts to the solution of the Lax conjecture for homogeneous hyperbolic polynomials in three variables, see [40]), in a somewhat weaker form (see also [57, Section 5] and [48]). Indeed, in [26] it was proven that $A_{1}$ and $A_{2}$ can be chosen to be real symmetric. The advantage of the approach here is that the proof uses factorizations of matrix polynomials (unlike the algebra-geometrical techniques used in [26]) making it especially suitable for computations.

\section{NoRM-CONSTRAINED DETERMINANTAL REPRESENTATIONS}

Throughout the paper we will assume that the polynomials are non-constant. Although one can adjust the definitions to include the case of $p \equiv 1$, it does not seem worth to do this. Given a non-constant bivariate polynomial $p$, its stability radius is defined as

$$
s(p):=\max \left\{r>0: p(z) \neq 0, z \in r \mathbb{D}^{2}\right\} .
$$

Thus $p$ is semi-stable if and only if $s(p) \geq 1$, and stable if and only if $s(p)>1$.

Theorem 2.1. Let $p\left(z_{1}, z_{2}\right)$, with $p(0,0)=1$, be a non-constant bivariate polynomial. Then $p$ admits a representation (1.1) with $n=\operatorname{deg} p$ and $\|K\|=s(p)^{-1}$.

Before we delve into the bivariate case, let us consider an alternative way to obtain (1.1) in the univariate case that does not require us to compute the roots of $p$. Let $p(z)=$ $p_{0}+\cdots+p_{n} z^{n}$ be a stable polynomial. Then the classical matrix theory says that $p$ is the characteristic polynomial of the associated companion matrix. Writing this in a form especially useful for our purposes, we have

$$
p(z)=p_{0} \operatorname{det}\left(I_{n}-z C_{p}\right),
$$

where

$$
C_{p}=\left[\begin{array}{cccc}
-\frac{p_{1}}{p_{0}} & 1 & & 0 \\
\vdots & & \ddots & \\
-\frac{p_{n-1}}{p_{0}} & 0 & & 1 \\
-\frac{p_{n}}{p_{0}} & 0 & \cdots & 0
\end{array}\right]
$$

As $p$ is stable, all the eigenvalues of $C_{p}$ lie in $\mathbb{D}$. Thus $C_{p}$ is similar to a strict contraction. For our purposes, it will suffice to find a similarity to a (not necessarily strict) contraction. To this end, we proceed by introducing the Bezoutian

$$
Q=A A^{*}-B^{*} B
$$

where

$$
A=\left[\begin{array}{ccc}
p_{0} & & \\
\vdots & \ddots & \\
p_{n-1} & \cdots & p_{0}
\end{array}\right], \quad B=\left[\begin{array}{ccc}
p_{n} & \cdots & p_{1} \\
& \ddots & \vdots \\
& & p_{n}
\end{array}\right]
$$


The Schur-Cohn criterion (see, e.g., [39, Section 13.5]) tells us that $p$ is stable if and only if $Q>0$. If we now factor $Q=P P^{*}$, with $P$ a square (and thus automatically invertible) matrix, then $K=P^{-1} C_{p} P$ is a contraction [59]. To see this, one shows that $\left[\begin{array}{cc}Q^{-1} & C_{p}^{*} Q^{-1} \\ Q^{-1} C_{p} & Q^{-1}\end{array}\right]$ and consequently $P\left(I-K^{*} K\right) P^{*}$ are positive semi-definite. Since the range of $K^{*}$ is contained in the range of $P^{*}$, it follows that $\|K\| \leq 1$. We now have the desired representation $p(z)=p_{0} \operatorname{det}\left(I_{n}-z K\right)$.

This alternative derivation of a representation (1.1) in a univariate case provides the basis for the construction of (1.1) in the bivariate case, where now the coefficients $p_{i}$ and the matrices $C_{p}, A, B, Q$, and $P$ will depend on one of the variables.

We will need the following two lemmata.

Lemma 2.2. If the polynomials $p_{1}$ and $p_{2}$ admit the representation (1.1) as in Theorem 2.1, then so does their product.

Proof. Although we formulate and prove the statement for bivariate polynomials, it is obviously extended to any number of variables.

Observe that if $n^{\prime}=\operatorname{deg} p_{1}$ and $n^{\prime \prime}=\operatorname{deg} p_{2}$, then $\operatorname{deg}\left(p_{1} p_{2}\right)=n^{\prime}+n^{\prime \prime}$ and $s\left(p_{1} p_{2}\right)=$ $\min \left\{s\left(p_{1}\right), s\left(p_{2}\right)\right\}$. If $p_{1}=\operatorname{det}\left(I_{\left|n^{\prime}\right|}-K_{1} Z_{n^{\prime}}\right)$ and $p_{2}=\operatorname{det}\left(I_{\left|n^{\prime \prime}\right|}-K_{2} Z_{n^{\prime}}\right)$, then $p_{1} p_{2}=$ $\operatorname{det}\left(I_{\left|n^{\prime}+n^{\prime \prime}\right|}-\tilde{K} \tilde{Z}\right)$, where $\tilde{K}=K_{1} \oplus K_{2}$ and $\tilde{Z}=Z_{n^{\prime}} \oplus Z_{n^{\prime \prime}}$. Applying a permutation $T$ which rearranges the coordinate variables on the diagonal of $Z$, we obtain that $p_{1} p_{2}=$ $\operatorname{det}\left(I_{\left|n^{\prime}+n^{\prime \prime}\right|}-K Z_{n^{\prime}+n^{\prime \prime}}\right)$ with $K=T \tilde{K} T^{-1}$ and $Z_{n^{\prime}+n^{\prime \prime}}=T \tilde{Z} T^{-1}$. Since $T$ is unitary, we obtain that

$$
\begin{aligned}
\|K\|=\|\tilde{K}\|=\max \left\{\left\|K_{1}\right\|,\right. & \left.\left\|K_{2}\right\|\right\} \\
& =\max \left\{s\left(p_{1}\right)^{-1}, s\left(p_{2}\right)^{-1}\right\}=\left(\min \left\{s\left(p_{1}\right), s\left(p_{2}\right)\right\}\right)^{-1}=s\left(p_{1} p_{2}\right)^{-1} .
\end{aligned}
$$

Recall that a polynomial $p$ is called irreducible if it has no nontrivial polynomial factors.

Lemma 2.3. Stable irreducible polynomials of a fixed bidegree with the constant term 1 are dense in the set of semi-stable polynomials of the same bidegree with the constant term 1

While we formulate and prove the statement here for bivariate polynomials, it can be obviously extended to any number of variables. We view polynomials of bidegree $\left(n_{1}, n_{2}\right)$ or less with constant term 1 as points in the coefficient space $\mathbb{C}^{N}$, where $N=\left(n_{1}+1\right)\left(n_{2}+1\right)-1$. Notice that if a sequence of polynomials in $\mathbb{C}^{N}$ converges to a polynomial of bidegree $\left(n_{1}, n_{2}\right)$, then the polynomials in the sequence will eventually have the same bidegree. Notice also that stable polynomials form an open set in $\mathbb{C}^{N}$.

Proof of Lemma 2.3. Observe that the set of reducible polynomials of bidegree $\left(n_{1}, n_{2}\right)$ or less with constant term 1 (the products of polynomials of smaller bidegrees) is a finite union of images of polynomial mappings $\mathbb{C}^{N^{\prime}} \times \mathbb{C}^{N^{\prime \prime}} \rightarrow \mathbb{C}^{N}$ where $N^{\prime}+N^{\prime \prime}<N$. Hence irreducible polynomials form an open dense subset of $\mathbb{C}^{N}$. Every semi-stable polynomial $p$ is a limit of stable dilations $p_{r}\left(z_{1}, z_{2}\right):=p\left(r z_{1}, r z_{2}\right)$ as $r \uparrow 1$. On the other hand, we can approximate $p_{r}$ by irreducible polynomials in $\mathbb{C}^{N}$. If an irreducible polynomial $q$ is sufficiently close to $p_{r}$, then $q$ is also stable. We conclude that every semi-stable polynomial in $\mathbb{C}^{N}$ 
can be approximated by stable irreducible polynomials; moreover, a semi-stable polynomial of bidegree $\left(n_{1}, n_{2}\right)$ can be approximated by stable irreducible polynomials of the same bidegree.

We will make use of the $1 \mathrm{D}$ system realization theory. A univariate matrix-valued rational function $f$ is said to have a (finite-dimensional) transfer-function realization if

$$
f(z)=D+C z(I-A z)^{-1} B
$$

for some complex matrix $\left[\begin{array}{cc}A & B \\ C & D\end{array}\right]$. A realization $(2.2)$ of $f$ is called minimal if the block $A$ is of minimal possible size. Every rational matrix-valued function $f$ which is analytic and contractive on $\mathbb{D}$ has a minimal realization [31]; moreover, the system matrix $\left[\begin{array}{ll}A & B \\ C & D\end{array}\right]$ of a minimal realization of $f$ can be chosen to be contractive [3].

Proof of Theorem 2.1. Without loss of generality, we may assume that $s(p)=1$, i.e., $p$ is semi-stable. Indeed, otherwise one proves the result for the semi-stable $q$, where $q(z)=$ $p(z s(p))$, resulting in a contraction $K_{q}$, and then put $K=K_{q} / s(p)$, to get the desired representation for $p$.

Next, if we can show the existence of a representation $\operatorname{det}\left(I_{|n|}-K Z_{n}\right)$, with $K$ a contraction, for a dense subset of semi-stable polynomials of bidegree $n$ with constant term 1 , then we are done. Indeed, if $p^{(j)}=\operatorname{det}\left(I_{|n|}-K^{(j)} Z_{n}\right)$, with $K^{(j)}$ a contraction, and $p^{(j)} \rightarrow p$, then $p=\operatorname{det}\left(I_{|n|}-K Z_{n}\right)$, where $K$ is a limit point of the sequence $\left\{K^{(j)}: j \in \mathbb{N}\right\}$ (which exists as the contractions in $\mathbb{C}^{|n| \times|n|}$ form a compact set). Thus, we are allowed to make some generic assumptions on $p$. For starters, by Lemma 2.3, we may assume that $p$ is stable and irreducible.

We now start the proof of the existence of a representation (1.1) with $n=\operatorname{deg} p$ and $\|K\| \leq 1$ for an irreducible stable polynomial $p$. Along the way, we make some other assumptions of genericity.

Expand $p$ in the powers of $z_{2}, p\left(z_{1}, z_{2}\right)=p_{0}\left(z_{1}\right)+\cdots+p_{n_{2}}\left(z_{1}\right) z_{2}^{n_{2}}$, and introduce the companion matrix

$$
\mathrm{C}\left(z_{1}\right)=\left[\begin{array}{cccc}
-\frac{p_{1}\left(z_{1}\right)}{p_{0}\left(z_{1}\right)} & 1 & & 0 \\
\vdots & & \ddots & \\
-\frac{p_{n_{2}-1}\left(z_{1}\right)}{p_{0}\left(z_{1}\right)} & 0 & & 1 \\
-\frac{p_{n_{2}}\left(z_{1}\right)}{p_{0}\left(z_{1}\right)} & 0 & \cdots & 0
\end{array}\right]
$$

and the triangular Toeplitz matrices

$$
\mathrm{A}\left(z_{1}\right)=\left[\begin{array}{ccc}
p_{0}\left(z_{1}\right) & & \\
\vdots & \ddots & \\
p_{n_{2}-1}\left(z_{1}\right) & \cdots & p_{0}\left(z_{1}\right)
\end{array}\right], \quad \mathrm{B}\left(z_{1}\right)=\left[\begin{array}{ccc}
p_{n_{2}}\left(z_{1}\right) & \cdots & p_{1}\left(z_{1}\right) \\
& \ddots & \vdots \\
& & p_{n_{2}}\left(z_{1}\right)
\end{array}\right]
$$

Form the Bezoutian $Q\left(z_{1}\right):=\mathrm{A}\left(z_{1}\right) \mathrm{A}\left(1 / \bar{z}_{1}\right)^{*}-\mathrm{B}\left(1 / \bar{z}_{1}\right)^{*} \mathrm{~B}\left(z_{1}\right)$.

Since the polynomial $p\left(z_{1}, \cdot\right)$ is stable for every $z_{1} \in \mathbb{T}$, we have that $Q\left(z_{1}\right)$ is positive definite for every $z_{1} \in \mathbb{T}\left[39\right.$, Section 13.5]. Then there exists a $n_{2} \times n_{2}$ matrix-valued polynomial $P\left(z_{1}\right)=P_{0}+\cdots+P_{n_{1}} z_{1}^{n_{1}}$ such that the factorization $Q\left(z_{1}\right)=P\left(z_{1}\right) P\left(z_{1}\right)^{*}$, 
$z_{1} \in \mathbb{T}$, holds and $P\left(z_{1}\right)$ is invertible for every $z_{1} \in \overline{\mathbb{D}}\left[51\right.$, 13]. Since $p_{0}\left(z_{1}\right)=p\left(z_{1}, 0\right) \neq 0$ for every $z_{1} \in \overline{\mathbb{D}}$, the rational matrix-valued function

$$
M\left(z_{1}\right):=P\left(z_{1}\right)^{-1} \mathrm{C}\left(z_{1}\right) P\left(z_{1}\right)
$$

is analytic on $\overline{\mathbb{D}}$. In fact, $M$ is also contractive there [59]. To see this, one shows that $\left[\begin{array}{cc}Q\left(z_{1}\right)^{-1} & \mathrm{C}\left(z_{1}\right)^{*} Q\left(z_{1}\right)^{-1} \\ Q\left(z_{1}\right)^{-1} \mathrm{C}\left(z_{1}\right) & Q\left(z_{1}\right)^{-1}\end{array}\right]$ and consequently $P\left(z_{1}\right)\left(I-M\left(z_{1}\right)^{*} M\left(z_{1}\right)\right) P\left(z_{1}\right)^{*}$ are positive semi-definite for $z_{1} \in \mathbb{T}$. Since the range of $M\left(z_{1}\right)^{*}$ is contained in the range of $P\left(z_{1}\right)^{*}$, it follows that $\left\|M\left(z_{1}\right)\right\| \leq 1$ for every $z_{1} \in \mathbb{T}$, and by the maximum principle, for every $z_{1} \in \overline{\mathbb{D}}$.

Claim: Generically, the only poles of $M$ are the zeros of $p_{0}$.

Proof of claim. We will first show that when $P$ has a zero at $z_{1}=a$ of geometric multiplicity 1 , the corresponding vector in the left kernel is a left eigenvector of $\mathrm{C}(a)$. Indeed, first observe that by analytic continuation, $Q\left(z_{1}\right)=P\left(z_{1}\right) P\left(1 / \bar{z}_{1}\right)^{*}$, where the analyticity domains of the rational matrix-valued functions on the two sides of the equality coincide. Then the zeros of $P$ are exactly the zeros of $Q$ that lie in $\mathbb{C} \backslash \overline{\mathbb{D}}$. Let $z_{1} \neq 0$. Observe that

$$
R\left(z_{1}\right):=\left[\begin{array}{ll}
\mathrm{A}\left(z_{1}\right) & \mathrm{B}\left(1 / \bar{z}_{1}\right)^{*} \\
\mathrm{~B}\left(z_{1}\right) & \mathrm{A}\left(1 / \bar{z}_{1}\right)^{*}
\end{array}\right]
$$

is the resultant for the polynomials $p\left(z_{1}, \cdot\right)$ and $g(\cdot)$, where

$$
g\left(z_{2}\right)=\overleftarrow{p}\left(z_{1}, z_{2}\right) / z_{1}^{n_{1}}=\overline{p_{n_{1}, n_{2}}} / z_{1}^{n_{1}}+\cdots+\overline{p_{00}} z_{2}^{n_{2}}
$$

Thus det $R\left(z_{1}\right)=0$ if and only if $p\left(z_{1}, z_{2}\right)=0=\overleftarrow{p}\left(z_{1}, z_{2}\right)$ for some $z_{2}$ (in the terminology of [19, 20]: $\left(z_{1}, z_{2}\right)$ is an intersecting zero). For such $z_{1}$ and $z_{2}$ we have that the row vector $v_{2 n_{2}-1}\left(z_{2}\right)$ is in the left kernel of $R\left(z_{1}\right)$; here

$$
v_{k}(z)=\left[\begin{array}{llll}
1 & z & \cdots & z^{k}
\end{array}\right]
$$

When $\overline{p_{0}}\left(1 / z_{1}\right) \neq 0$, we have that $\mathrm{A}\left(1 / \overline{z_{1}}\right)^{*}$ is invertible, and

$$
\left[\begin{array}{lll}
v_{n_{2}-1}\left(z_{2}\right) & z_{2}^{n_{2}} v_{n_{2}-1}\left(z_{2}\right)
\end{array}\right]\left[\begin{array}{ll}
\mathrm{A}\left(z_{1}\right) & \mathrm{B}\left(1 / \bar{z}_{1}\right)^{*} \\
\mathrm{~B}\left(z_{1}\right) & \mathrm{A}\left(1 / \bar{z}_{1}\right)^{*}
\end{array}\right]=0
$$

implies

$$
v_{n_{2}-1}\left(z_{2}\right) Q\left(z_{1}\right)=0 \text {. }
$$

Notice that we used that $\mathrm{A}\left(w_{1}\right)^{*}$ and $\mathrm{B}\left(w_{2}\right)$ commute as they are both upper triangular Toeplitz matrices. Thus when $P$ has a zero at $z_{1}=a$ of geometric multiplicity 1 , its vector in the left kernel is $v_{n_{2}-1}\left(z_{2}\right)$, where $\left(a, z_{2}\right)$ is an intersecting zerd2. It is now straightforward to check that $v_{n_{2}-1}\left(z_{2}\right)$ is a left eigenvector of $\mathrm{C}(a)$ corresponding to the eigenvalue $1 / z_{2}$.

To show that the only poles of $M$ are the zeros of $p_{0}$, we observe that the only other possible source of poles of $M$ would be the zeros of $P$. Assuming (a generic condition!) that such a zero $a$ has multiplicity 1 and is not a zero of $p_{0}$, we obtain that

$$
P\left(z_{1}\right)=P_{0}+P_{1}\left(z_{1}-a\right)+\cdots+P_{n_{1}}\left(z_{1}-a\right)^{n_{1}}, \quad P\left(z_{1}\right)^{-1}=S_{-1} /\left(z_{1}-a\right)+S_{0}+S_{1}\left(z_{1}-a\right)+\cdots,
$$

${ }^{2}$ Alternatively, one may use the formula

$$
\frac{p\left(z_{1}, z_{2}\right) \overline{p\left(1 / \overline{z_{1}}, z_{2}\right)}-\overleftarrow{p}\left(z_{1}, z_{2}\right) \overline{\overleftarrow{p}\left(1 / \overline{z_{1}}, z_{2}\right)}}{1-\left|z_{2}\right|^{2}}=v_{n_{2}-1}\left(z_{2}\right) Q\left(z_{1}\right) v_{n_{2}-1}\left(z_{2}\right)^{*}
$$

to come to the same conclusion. This formula can be easily checked by hand, but also appears in many sources; see, e.g., 30, Section 4]. 
where $\operatorname{dim} \operatorname{Ker} P_{0}=1$ and $\operatorname{rank} S_{-1}=1$ (see, e.g., [8, Chapter II]). In addition, as $P\left(z_{1}\right)$ and $P\left(z_{1}\right)^{-1}$ multiply to $I_{n_{2}}$, we have $P_{0} S_{-1}=0=S_{-1} P_{0}$. By the result of the previous paragraph, we must have that $S_{-1}=w v_{n_{2}-1}\left(z_{2}\right)$, for some column vector $w$. But then we have that

$$
M\left(z_{1}\right)=P\left(z_{1}\right)^{-1} \mathrm{C}\left(z_{1}\right) P\left(z_{1}\right)=S_{-1} \mathrm{C}(a) P_{0} /\left(z_{1}-a\right)+G\left(z_{1}\right),
$$

where $G\left(z_{1}\right)$ is analytic in a neighborhood of $a$. Since

$$
v_{n_{2}-1}\left(z_{2}\right) \mathrm{C}(a) P_{0}=\left(1 / z_{2}\right) v_{n_{2}-1}\left(z_{2}\right) P(a)=0,
$$

we have $S_{-1} \mathrm{C}(a) P_{0}=0$, and thus $M\left(z_{1}\right)$ does not have a pole at $a$. This proves the claim.

We assume now for $p$ the generic assumptions above and, in addition, the assumption that $p_{n_{2}}$ is of degree $n_{1}$ and is coprime with $p_{0}$, which is also generic. Then the McMillan degree of $\mathrm{C}$, and hence, of $\mathrm{M}$, is $n_{1}$, therefore there exists a minimal contractive realization of $M$ with $A$ a $n_{1} \times n_{1}$ matrix:

$$
M\left(z_{1}\right)=D+C z_{1}\left(I_{n_{1}}-A z_{1}\right)^{-1} B
$$

(see [8, Section 4.2] or [5, Sections 4.1,4.2] for the notion of the McMillan degree of a rational matrix-valued function and its equality to the size of a minimal realization of the function). We have

$$
p\left(z_{1}, z_{2}\right)=p_{0}\left(z_{1}\right) \operatorname{det}\left(I_{n_{2}}-M\left(z_{1}\right) z_{2}\right)=\frac{p_{0}\left(z_{1}\right)}{\operatorname{det}\left(I_{n_{1}}-z_{1} A\right)} \operatorname{det}\left(I_{|n|}-\left[\begin{array}{cc}
A & B \\
C & D
\end{array}\right]\left[\begin{array}{cc}
z_{1} I_{n_{1}} & 0 \\
0 & z_{2} I_{n_{2}}
\end{array}\right]\right) \text {. }
$$

As $\operatorname{det}\left(I_{n_{1}}-z_{1} A\right)$ is the denominator of the coprime fraction representation of $\operatorname{det} M[5$, Section 4.2], it follows that $p_{0}\left(z_{1}\right)=\operatorname{det}\left(I_{n_{1}}-z_{1} A\right)$. This proves that

$$
p\left(z_{1}, z_{2}\right)=\operatorname{det}\left(I_{|n|}-\left[\begin{array}{cc}
A & B \\
C & D
\end{array}\right]\left[\begin{array}{cc}
z_{1} I_{n_{1}} & 0 \\
0 & z_{2} I_{n_{2}}
\end{array}\right]\right),
$$

and we are done.

Notice that the proof outlines a procedure to find a representation (1.1). Let us try this out on a simple example.

Example 2.4. Let $p\left(z_{1}, z_{2}\right)=1+a z_{1}+b z_{2}$, where $a+b<1$ and $a, b>0$. Then $p_{0}\left(z_{1}\right)=1+a z_{1}$ and $p_{1}\left(z_{1}\right)=b$. We have

$$
M\left(z_{1}\right)=\mathrm{C}\left(z_{1}\right)=-\frac{b}{1+a z_{1}}=-b+\sqrt{a b} z_{1}\left(1+a z_{1}\right)^{-1} \sqrt{a b}
$$

and obtain the representation $p\left(z_{1}, z_{2}\right)=\operatorname{det}\left(I_{2}-\left[\begin{array}{cc}-a & \sqrt{a b} \\ \sqrt{a b} & -b\end{array}\right]\left[\begin{array}{cc}z_{1} & 0 \\ 0 & z_{2}\end{array}\right]\right)$ with a contraction $\left[\begin{array}{cc}-a & \sqrt{a b} \\ \sqrt{a b} & -b\end{array}\right]$.

Kummert [36, 35, 37] proved Theorem 2.1 for bivariate scattering Schur polynomials. He first constructed for such a polynomial $p$ a $2 D$ Givone-Roesser system realization [21] of $f=\overleftarrow{p} / p$

$$
f=D+C Z_{n}\left(I_{|n|}-A Z_{n}\right)^{-1} B
$$


with the complex $(|n|+1) \times(|n|+1)$ matrix $\left[\begin{array}{cc}A & B \\ C & D\end{array}\right]$ being unitary, and then wrote it as

$$
\frac{\overleftarrow{p}}{p}=\frac{\operatorname{det}\left[\begin{array}{cc}
I_{|n|}-A Z_{n} & B \\
-C Z_{n} & D
\end{array}\right]}{\operatorname{det}\left(I_{|n|}-A Z_{n}\right)}
$$

Since the fraction representation on the left-hand side is coprime and the bidegree of the polynomial in the denominator of the fraction on the right-hand side is less than or equal $n=\left(n_{1}, n_{2}\right)$ (in the componentwise sense), the denominators must be equal:

$$
p=\operatorname{det}\left(I_{|n|}-A Z_{n}\right) .
$$

Since $A$ is a contraction, Theorem 2.1 follows for this case, with $K=A$. We also remark that in this construction $K$ has all singular values, except one, equal to 1.

Let us note that the existence of 2D Givone-Roesser unitary system realizations was proved by Agler [1] for a much more general class of contractive analytic operator-valued functions on the bidisk $\mathbb{D}^{2}$, however the (unitary) system matrix in such a realization has, in general, infinite-dimensional Hilbert-space operator blocks (in particular, $A$ is a contraction on an infinite-dimensional Hilbert space). Kummert's result in the special case of scalar rational inner functions is sharper in the sense that it provides a concrete finite-dimensional unitary realization of the smallest possible size. We also remark that an alternative construction of a finite-dimensional Givone-Roesser unitary system realization for matrix-valued rational inner functions of two-variables is given in [6].

The general case of Theorem 2.1 can also be deduced from the special case of scattering Schur polynomials, since the latter is a dense set in the space of all bivariate polynomials of bidegree $n=\left(n_{1}, n_{2}\right)$ with the constant term 1 , and the approximation argument as in our proof of Theorem 2.1 works. In view of Lemma 2.3, it suffices to prove the following proposition.

Proposition 2.5. Every stable irreducible polynomial is scattering Schur.

Proof. We will prove the statement here for bivariate polynomials, but it can obviously be extended to any number of variables.

Suppose $p$ is a stable irreducible bivariate polynomial and is not scattering Schur. Then it must divide $\overleftarrow{p}$

If $p$ is a nontrivial polynomial depending only on one of the variables, say $z_{1}$, then it has the form $p\left(z_{1}, z_{2}\right)=a\left(z_{1}-z_{0}\right)$, with $a \in \mathbb{C} \backslash\{0\}$ and $z_{0} \in \mathbb{C} \backslash \overline{\mathbb{D}}$, and $\overleftarrow{p}\left(z_{1}, z_{2}\right)=-\bar{a} \overline{z_{0}}\left(z_{1}-1 / \overline{z_{0}}\right)$, which is impossible.

If $p$ depends on both $z_{1}$ and $z_{2}$, then it is possible to fix one of the variables, say $z_{2}=\lambda$, on the unit circle $\mathbb{T}$ so that $q\left(z_{1}\right)=p\left(z_{1}, \lambda\right)$ is a nontrivial polynomial in $z_{1}$. Then $q$ has no zeros in $\overline{\mathbb{D}}$ and, since the polynomial $q\left(z_{1}\right)$ divides $\overleftarrow{p}\left(z_{1}, \lambda\right), q$ can not have zeros in $\mathbb{C} \backslash \overline{\mathbb{D}}$ a contradiction to the Fundamental Theorem of Algebra.

Thus, $p$ is scattering Schur.

We note that another stability criterion for bivariate polynomials was established in [33, Theorem 1.1]. Namely, it was shown that $p$ is stable if and only if

$$
\left|p\left(z_{1}, z_{2}\right)\right|^{2}-\left|\overleftarrow{p}\left(z_{1}, z_{2}\right)\right|^{2} \underset{8}{c} \underset{8}{\left(1-\left|z_{1}\right|^{2}\right)}\left(1-\left|z_{2}\right|^{2}\right), z_{1}, z_{2} \in \mathbb{D}
$$


for some $c>0$. Moreover, when $p$ is stable, one may choose

$$
c=4 \pi\left(\int_{0}^{2 \pi} \int_{0}^{2 \pi} \frac{1}{\left|p\left(e^{i \theta}, e^{i \psi}\right)\right|^{2}} d \theta d \psi\right)^{-1} .
$$

A $d$-variable generalization of (2.6) may be found in [10, Theorem 5.1].

\section{The CASE OF SELF-REVERSIVE POLYNOMIALS}

Given a semi-stable polynomial $p$, one has the factorization $p=u s$, where $u$ is a semistable self-reversive polynomial and $s$ is a scattering Schur polynomial [9, Theorem 4]. In the case where $p$ is semi-stable and self-reversive, the factor $s$ is a constant. Our next theorem specializes the result of Theorem 2.1 to this case. We first establish several equivalent conditions for a semi-stable polynomial to be self-reversive; while we formulate and prove the next proposition for bivariate polynomials, it is clear that it extends to any number of variables.

Proposition 3.1. Let $p$ be a semi-stable bivariate polynomial of bidegree $n=\left(n_{1}, n_{2}\right)$ with $p(0,0)=1$; then the following statements are equivalent:

(i) $p$ is self-reversive up to a unimodular constant;

(ii) the coefficient of $z_{1}^{n_{1}} z_{2}^{n_{2}}$ in $p$ is unimodular;

(iii) if $\left(z_{1}, z_{2}\right) \in \mathbb{T}^{2}$, the one-variable polynomial $t \mapsto p\left(t z_{1}, t z_{2}\right)$ has all its zeros on $\mathbb{T}$.

Proof. (i) $\Rightarrow$ (ii) is obvious, since for a bivariate polynomial $p$ of bidegree $n=\left(n_{1}, n_{2}\right)$, the free term of $\overleftarrow{p}$ equals the conjugate of the coefficient of $z_{1}^{n_{1}} z_{2}^{n_{2}}$ in $p$.

(ii) $\Rightarrow$ (iii) Let $p\left(z_{1}, z_{2}\right)=\sum_{j=0}^{r} p_{j}\left(z_{1}, z_{2}\right), p_{0}\left(z_{1}, z_{2}\right)=1$, be the expansion of $p$ in homogeneous polynomials. Then

$$
p_{\left(z_{1}, z_{2}\right)}(t):=p\left(t z_{1}, t z_{2}\right)=\sum_{j=0}^{r} p_{j}\left(z_{1}, z_{2}\right) t^{j} .
$$

If the coefficient $p_{n_{1}, n_{2}}$ of $z_{1}^{n_{1}} z_{2}^{n_{2}}$ is unimodular, then $r=n_{1}+n_{2}$ and $p_{r}\left(z_{1}, z_{2}\right)=p_{n_{1}, n_{2}} z_{1}^{n_{1}} z_{2}^{n_{2}}$. For $\left(z_{1}, z_{2}\right) \in \mathbb{T}^{2}$, we can write

$$
p_{\left(z_{1}, z_{2}\right)}(t)=\left(1-a_{1}\left(z_{1}, z_{2}\right) t\right) \cdots\left(1-a_{r}\left(z_{1}, z\right) t\right),
$$

where $1 / a_{1}\left(z_{1}, z_{2}\right), \ldots, 1 / a_{r}\left(z_{1}, z_{2}\right)$ are the roots of $p_{\left(z_{1}, z_{2}\right)}$ counting multiplicities. Because of semi-stability, $\left|a_{i}\left(z_{1}, z_{2}\right)\right| \geq 1$; but $p_{r}\left(z_{1}, z_{2}\right)=(-1)^{r} a_{1}\left(z_{1}, z_{2}\right) \cdots a_{r}\left(z_{1}, z_{2}\right)$ is unimodular, hence $\left|a_{i}\left(z_{1}, z_{2}\right)\right|=1$.

(iii) $\Rightarrow$ (i) Let $p_{\left(z_{1}, z_{2}\right)}$ be as in (3.1). By the assumption, for every $\left(z_{1}, z_{2}\right) \in \mathbb{T}^{2}$ the polynomial $p_{\left(z_{1}, z_{2}\right)}$ is self-reversive up to a unimodular constant, hence $p_{r}\left(z_{1}, z_{2}\right)$ is either zero or unimodular. Since the polynomial $p_{r}$ is nonzero, it is not identically zero on $\mathbb{T}^{2}$ (e.g., by the uniqueness principle for bivariate analytic functions). By continuity, $p_{r}\left(z_{1}, z_{2}\right)$ is unimodular, and thus $\operatorname{deg} p_{\left(z_{1}, z_{2}\right)}=r$ for every $\left(z_{1}, z_{2}\right) \in \mathbb{T}^{2}$. It follows (e.g., by Rudin's characterization of rational inner functions [53, Theorem 5.2.5]) that $p_{r}$ is a monomial:

$$
p_{r}\left(z_{1}, z_{2}\right)=p_{m_{1}, m_{2}} z_{1}^{m_{1}} z_{2}^{m_{2}}
$$

with $\left(m_{1}, m_{2}\right) \leq\left(n_{1}, n_{2}\right),|m|=r$, and $\left|p_{m_{1}, m_{2}}\right|=1$. 
Now, the fact that for $\left(z_{1}, z_{2}\right) \in \mathbb{T}^{2}$ the polynomial $p_{\left(z_{1}, z_{2}\right)}$ is self-reversive up to a unimodular constant implies that

$$
\overline{p_{r-j}\left(z_{1}, z_{2}\right)}=\overline{p_{r}\left(z_{1}, z_{2}\right)} p_{j}\left(z_{1}, z_{2}\right)
$$

for $\left(z_{1}, z_{2}\right) \in \mathbb{T}^{2}$ and $j=0, \ldots, r$, and therefore by analytic continuation

$$
\overline{p_{r-j}\left(\frac{1}{\bar{z}_{1}}, \frac{1}{\bar{z}_{2}}\right)}=\overline{p_{r}\left(\frac{1}{\bar{z}_{1}}, \frac{1}{\bar{z}_{2}}\right)} p_{j}\left(z_{1}, z_{2}\right)
$$

for all $z_{1}, z_{2} \neq 0$ and $j=0, \ldots, r$. It follows that

$$
\overline{p\left(\frac{1}{\bar{z}_{1}}, \frac{1}{\bar{z}_{2}}\right)}=\overline{p_{r}\left(\frac{1}{\bar{z}_{1}}, \frac{1}{\bar{z}_{2}}\right)} p\left(z_{1}, z_{2}\right)
$$

and finally, since $p_{r}\left(z_{1}, z_{2}\right)=p_{m_{1}, m_{2}} z_{1}^{m_{1}} z_{2}^{m_{2}}$,

$$
z_{1}^{m_{1}} z_{2}^{m_{2}} \overline{p\left(\frac{1}{\bar{z}_{1}}, \frac{1}{\bar{z}_{2}}\right)}=\bar{p}_{m_{1}, m_{2}} p\left(z_{1}, z_{2}\right) .
$$

Comparing the degrees of $z_{1}$ and $z_{2}$ we see that $n_{1}=m_{1}, n_{2}=m_{2}$, and $p$ is self-reversive up to the unimodular constant $\bar{p}_{m_{1}, m_{2}}$.

Theorem 3.2. Let the bivariate polynomial $p$ of bidegree $n=\left(n_{1}, n_{2}\right) \neq(0,0)$, with $p(0,0)=$ 1 , be semi-stable. Then $p$ is self-reversive up to a unimodular constant if and only if $p$ admits a representation (1.1) with $n=\operatorname{deg} p$ and $K$ unitary.

Proof. The proof in one direction is immediate. If $p=\operatorname{det}\left(I_{|n|}-K Z_{n}\right)$, with $K$ unitary and $n=\operatorname{deg} p$, then

$$
\begin{aligned}
\overleftarrow{p}(z)=z^{n} \operatorname{det}\left(I_{|n|}-\bar{K} Z_{n}^{-1}\right)=z^{n} \operatorname{det}\left(I_{|n|}-Z_{n}^{-1} K^{*}\right)=\operatorname{det} Z_{n} \operatorname{det}\left(I_{|n|}-Z_{n}^{-1} K^{*}\right) \\
=\operatorname{det}\left(Z_{n}-K^{*}\right)=\operatorname{det}\left(-K^{*}\right) \operatorname{det}\left(I_{|n|}-K Z_{n}\right)=\alpha p(z)
\end{aligned}
$$

with $\alpha=\operatorname{det}\left(-K^{*}\right) \in \mathbb{T}$.

Conversely, assume that $p$ is self-reversive up to a unimodular constant, or equivalently (by Proposition 3.1) that the coefficient of $z_{1}^{n_{1}} z_{2}^{n_{2}}$ in $p$ is unimodular. By Theorem 2.1, $p$ has a representation (1.1) with a contractive $K$. Observe that the modulus of the coefficient of $z_{1}^{n_{1}} z_{2}^{n_{2}}$ equals $|\operatorname{det} K|$, which in turn equals the product of the singular values of $K$. As $|\operatorname{det} K|=1$, all singular values of $K$ must be equal to 1 , yielding that $K$ is unitary.

We notice that the procedure outlined in the proof of Theorem 2.1 to find a representation (1.1) does not work for self-reversive polynomials as the Bezoutian $Q$ is 0 , and a limiting process (as in the beginning of the proof of Theorem 2.1) is necessary.

The non-trivial direction of Theorem 3.2 was previously established directly in [18] (generalizing the determinantal representations considered in [2]). More precisely, [18, Theorem 10.5] establishes that a bivariate polynomial $p$ of bidegree $\left(n_{1}, n_{2}\right)$ with $p(0,0)=1$, having no irreducible factors of the form $\alpha z_{1}+\beta, \alpha, \beta \in \mathbb{C}$, admits a representation (1.1) with $n=\operatorname{deg} p$ and $K$ unitary, provided that $p\left(z_{1}, z_{2}\right)=0$ and $\left|z_{1}\right|=1$ imply $\left|z_{2}\right|=1$, and $p(t, 0)$ is a stable polynomial in $t$ (in the notation of [18, Theorem 10.5], this is the special case $n_{2}=0$, so $\left.n_{1}=n\right)$. It suffices therefore to notice the following proposition.

Proposition 3.3. Let $p$ be a bivariate polynomial of bidegree $\left(n_{1}, n_{2}\right)$ with $p(0,0)=1$, having no irreducible factors of the form $\alpha z_{1}+\beta, \alpha, \beta \in \mathbb{C}$. Then the following are equivalent: 
(i) $p$ is semi-stable and self-reversive up to a unimodular constant;

(ii) $p\left(z_{1}, z_{2}\right)=0$ and $\left|z_{1}\right|=1$ imply $\left|z_{2}\right|=1$, and $p(t, 0)$ is stable in $t$.

Proof. Both (i) and (ii) are inherited by the irreducible factors of $p$, so we may assume without loss of generalty that $p$ is irreducible with $n_{2}>0$. We denote by $X$ the desingularizing Riemann surface of the projective closure of the zero set $Z_{p}$ of $p$ in $\mathbb{C}^{2}$, and we abuse the notation by letting $z_{1}$ and $z_{2}$ denote both the coordinates in $\mathbb{C}^{2}$ and the corresponding meromorphic functions on $X$.

(i) $\Longrightarrow$ (ii) The second condition in (ii) is obvious. Assume by contradiction that $p\left(z^{0}\right)=0, z^{0}=\left(z_{1}^{0}, z_{2}^{0}\right),\left|z_{1}^{0}\right|=1,\left|z_{2}^{0}\right| \neq 1$. Since $p$ is self-reversive, we may assume that $\left|z_{2}^{0}\right|<1$. Let $\xi^{0} \in X$ lie above $z^{0}$. Since $z_{1}$ is a non-constant meromorphic function on $X$, it is an open mapping, hence there exists $\xi \in X$ near $\xi^{0}$ such that $\left|z_{1}(\xi)\right|<1$ and $\left|z_{2}(\xi)\right|<1$ contradicting the semi-stability of $p$.

(ii) $\Longrightarrow$ (i) [18, Lemma 10.6] shows that $p$ is self-reversive up to a unimodular constant. Since $p$ is self-reversive, $X$ is endowed with an anti-holomorphic involution $\tau$, and the coordinate functions $z_{1}$ and $z_{2}$ on $X$ are unimodular meromorphic functions (i.e., $1 / \overline{z_{1}(\tau(\xi))}=z_{1}(\xi)$ and similarly for $\left.z_{2}\right)$. The condition $p\left(z_{1}, z_{2}\right)=0$ and $\left|z_{1}\right|=1$ imply $\left|z_{2}\right|=1$ implies further that $X$ is of dividing type, i.e., $X=X_{+} \cup X_{\mathbb{R}} \cup X_{-}$, where the union is disjoint and the three subsets in the decomposition are the preimages under $z_{1}$ of $\mathbb{D}, \mathbb{T}$, and the exterior of the unit disc (including $\infty$ ), respectively. Now, $p(t, 0)$ is stable in $t$ means simply that all the zeroes of $z_{2}$ on $X$ lie in $X_{-}$, therefore $z_{2}$ is an inverse of an inner function on $X_{+}$. So: $z_{1}$ is inner and $1 / z_{2}$ is inner on $X_{+}$, implying that $p$ is semi-stable.

We sketch now the construction of [18] as adapted to our case, which is both simpler and more feasible from a computational viewpoint than the general situation considered there. Suppose $p \in \mathbb{C}\left[z_{1}, z_{2}\right]$ is a semi-stable self-reversive polynomial with $p(0,0)=1$. By Lemma 2.2, it suffices to assume that $p$ is irreducible and obtain a representation (1.1) with $\operatorname{deg} p=\left(n_{1}, n_{2}\right)$ and $K$ unitary for this case. We assume that $n_{2}>0$, the case $n_{2}=0$ being trivial. It follows [18, Lemma 10.7] that the polynomial $\overleftarrow{\partial p / \partial z_{2}}$ is semi-stable and $\frac{z_{2} \partial p / \partial z_{2}}{\partial p / \partial z_{2}}$ is a coprime fraction representation of a rational inner function. Then it is well known (see [37, 19, 6, 33]) that there exist bivariate polynomials $A_{1}, \ldots, A_{n_{1}}$ of bidegree $\left(n_{1}-1, n_{2}\right)$ or less, and bivariate polynomials $B_{1}, \ldots, B_{n_{2}}$ of bidegree $\left(n_{1}, n_{2}-1\right)$ or less, not all of them equal 0 , such that

$$
\begin{aligned}
& \overleftarrow{\frac{\partial p}{\partial z_{2}}}\left(z_{1}, z_{2}\right) \overline{\overleftarrow{\partial p}}\left(w_{1}, w_{2}\right)-z_{2} \overline{w_{2}} \frac{\partial p}{\partial z_{2}}\left(z_{1}, z_{2}\right) \overline{\frac{\partial p}{\partial z_{2}}\left(w_{1}, w_{2}\right)} \\
& =\left(1-z_{1} \overline{w_{1}}\right) \sum_{i=1}^{n_{1}} A_{i}\left(z_{1}, z_{2}\right) \overline{A_{i}\left(w_{1}, w_{2}\right)}+\left(1-z_{2} \overline{w_{2}}\right) \sum_{j=1}^{n_{2}} B_{j}\left(z_{1}, z_{2}\right) \overline{B_{j}\left(w_{1}, w_{2}\right)}
\end{aligned}
$$

(this replaces a more general decomposition with "negative squares" provided by [18, Theorem 10.1]); furthermore, these polynomials can be found using semidefinite programming software. It is straightforward to verify the identity $n_{2} p=\frac{\overleftarrow{\partial p}}{\partial z_{2}}+z_{2} \frac{\partial p}{\partial z_{2}}$, which implies that the left-hand side of (3.2) is equal to

$$
n_{2}^{2} p\left(z_{1}, z_{2}\right) \overline{p\left(w_{1}, w_{2}\right)}-n_{2} z_{2} \frac{\partial p}{\partial z_{2}}\left(z_{1}, z_{2}\right) \overline{p\left(w_{1}, w_{2}\right)}-n_{2} p\left(z_{1}, z_{2}\right) \overline{w_{2} \frac{\partial p}{\partial z_{2}}\left(w_{1}, w_{2}\right)} .
$$


When both $\left(z_{1}, z_{2}\right)$ and $\left(w_{1}, w_{2}\right)$ lie in the zero set $Z_{p}$ of the polynomial $p$, this expression and, thus, the left-hand side of (3.2) are equal to 0 . Then we use the standard "lurking isometry" argument. We first rewrite the equality (3.2) restricted to $Z_{p} \times Z_{p}$ as

$$
\left[\begin{array}{l}
A\left(w_{1}, w_{2}\right) \\
B\left(w_{1}, w_{2}\right)
\end{array}\right]^{*}\left[\begin{array}{l}
A\left(z_{1}, z_{2}\right) \\
B\left(z_{1}, z_{2}\right)
\end{array}\right]=\left[\begin{array}{l}
w_{1} A\left(w_{1}, w_{2}\right) \\
w_{2} B\left(w_{1}, w_{2}\right)
\end{array}\right]^{*}\left[\begin{array}{l}
z_{1} A\left(z_{1}, z_{2}\right) \\
z_{2} B\left(z_{1}, z_{2}\right)
\end{array}\right]
$$

where $A\left(z_{1}, z_{2}\right):=\operatorname{col}_{i=1, \ldots, n_{1}}\left[A_{i}\left(z_{1}, z_{2}\right)\right]$ and $B\left(z_{1}, z_{2}\right):=\operatorname{col}_{i=1, \ldots, n_{2}}\left[B_{i}\left(z_{1}, z_{2}\right)\right]$. Then we observe that this identity uniquely determines an isometry

$$
T: \operatorname{span}\left\{\left[\begin{array}{l}
z_{1} A\left(z_{1}, z_{2}\right) \\
z_{2} B\left(z_{1}, z_{2}\right)
\end{array}\right]:\left(z_{1}, z_{2}\right) \in Z_{p}\right\} \rightarrow \operatorname{span}\left\{\left[\begin{array}{l}
A\left(z_{1}, z_{2}\right) \\
B\left(z_{1}, z_{2}\right)
\end{array}\right]:\left(z_{1}, z_{2}\right) \in Z_{p}\right\},
$$

defined on generating vectors by

$$
T:\left[\begin{array}{l}
z_{1} A\left(z_{1}, z_{2}\right) \\
z_{2} B\left(z_{1}, z_{2}\right)
\end{array}\right] \mapsto\left[\begin{array}{l}
A\left(z_{1}, z_{2}\right) \\
B\left(z_{1}, z_{2}\right)
\end{array}\right]
$$

and then extended by linearity. We shall see a posteriori that in fact the span of the vectors on the right-hand side is all of $\mathbb{C}^{|n|}$, so that the isometry $T$ is a unitary mapping of $\mathbb{C}^{|n|}$ onto itself. At any rate, $T$ can be extended to a unitary mapping $K$ of $\mathbb{C}^{|n|}$ onto itself, i.e., to a $|n| \times|n|$ unitary matrix.

The nonzero polynomial $P=\left[\begin{array}{l}A \\ B\end{array}\right] \in \mathbb{C}^{|n|}\left[z_{1}, z_{2}\right]$ does not vanish identically on $Z_{p}$. Indeed, Bézout's theorem [15, p. 112] says that two bivariate polynomials with no common factors can have at most a finite number of common zeros equal to the product of total degrees of the polynomials. Therefore, if $P$ vanishes identically on $Z_{p}$, then the irreducible polynomial $p$ should divide every component of $P$, but since these components, $A_{i}, i=1, \ldots, n_{1}$, and $B_{j}$, $j=1, \ldots, n_{2}$, are polynomials of smaller bidegree than $p$, this is impossible. Moreover, the set $Z_{p} \backslash Z_{P}$ is Zariski relatively open and dense in $Z_{p}$. Since the polynomial $q=\operatorname{det}\left(I_{|n|}-K Z_{n}\right)$ vanishes on this set, it vanishes on $Z_{p}$ as well. Applying Bézout's theorem again, we see that $p$ divides $q$. Since $\operatorname{deg} q=\operatorname{deg} p=n$ and $p(0)=q(0)=1$, we must have $p=q$, i.e., $p$ has a representation (1.1) with $n=\operatorname{deg} p$ and $K$ unitary. This provides an alternative proof of the non-trivial direction in Theorem 3.2 .

We notice that the restriction of $P=\left[\begin{array}{l}A \\ B\end{array}\right] \in \mathbb{C}^{|n|}\left[z_{1}, z_{2}\right]$ to $Z_{p}$ is a section of the kernel bundle of the determinantal representation $\bar{I}_{|n|}-K Z_{n}$ of the irreducible polynomial $p$, see [55, 32]. It follows (essentially since such a section is generated by the columns of the adjoint matrix $\left.\operatorname{adj}\left(I_{|n|}-K Z_{n}\right)\right)$ that the entries of the restriction of $P$ to $Z_{p}$ are linearly independent, in other words there exists no nonzero $c \in \mathbb{C}^{1 \times|n|}$ such that $c P\left(z_{1}, z_{2}\right)=0$ for all $\left(z_{1}, z_{2}\right) \in Z_{p}$. Therefore the span of $P\left(z_{1}, z_{2}\right),\left(z_{1}, z_{2}\right) \in Z_{p}$, is all of $\mathbb{C}^{|n|}$, so that the isometry $T=K$ is already a unitary mapping of $\mathbb{C}^{|n|}$ onto itself and no extension is needed.

We illustrate this on the following example.

Example 3.4. Let $p\left(z_{1}, z_{2}\right)=1-z_{1} z_{2}-\frac{1}{2} z_{1}^{2}-\frac{1}{2} z_{2}^{2}+z_{1}^{2} z_{2}^{2}$, so that $\operatorname{deg} p=(2,2)$. We compute

$$
\frac{\partial p}{\partial z_{2}}\left(z_{1}, z_{2}\right)=-z_{1}-z_{2}+2 z_{1}^{2} z_{2}, \quad \overleftarrow{\partial p}\left(z_{1}, z_{2}\right)=2-z_{1} z_{2}-z_{1}^{2}
$$


and find (using semidefinite programming software)

$$
\begin{gathered}
A_{1}\left(z_{1}, z_{2}\right)=\sqrt{2}\left(1-z_{1} z_{2}\right), \quad A_{2}\left(z_{1}, z_{2}\right)=z_{1}-z_{2} \\
B_{1}\left(z_{1}, z_{2}\right)=\sqrt{2}\left(1-z_{1}^{2}\right), \quad B_{2}\left(z_{1}, z_{2}\right)=z_{1}+z_{2}-2 z_{1}^{2} z_{2},
\end{gathered}
$$

so that $(\underline{3.2})$ holds. Taking the zeros $(0, \sqrt{2}),(\sqrt{2}, 0),\left(\frac{1}{2},-1+\frac{3}{\sqrt{2}}\right),\left(-1+\frac{3}{\sqrt{2}}, \frac{1}{2}\right)$, we find that the unitary $K=T$ is the matrix

$$
K=\frac{1}{\sqrt{2}}\left[\begin{array}{cccc}
0 & 1 & 0 & 1 \\
1 & 0 & -1 & 0 \\
0 & -1 & 0 & 1 \\
1 & 0 & 1 & 0
\end{array}\right]
$$

One can easily check that $p\left(z_{1}, z_{2}\right)=\operatorname{det}\left(I_{4}-K Z_{(2,2)}\right)$.

\section{REAL-ZERo POLYNOMIALS AND SELF-ADJOINT DETERMINANTAL REPRESENTATIONS}

We consider bivariate real-zero polynomials, which are polynomials $p \in \mathbb{R}\left[x_{1}, x_{2}\right]$ with the property that for every $\left(x_{1}, x_{2}\right) \in \mathbb{R}^{2}$ the one-variable polynomial $p_{\left(x_{1}, x_{2}\right)}(t):=p\left(t x_{1}, t x_{2}\right)$ has only real zeros. In [26, Theorem 2.2] it was shown that every real-zero polynomial $p$ with $p(0,0)=1$ may be represented as

$$
p\left(x_{1}, x_{2}\right)=\operatorname{det}\left(I+x_{1} A_{1}+x_{2} A_{2}\right),
$$

where $A_{1}, A_{2} \in \mathbb{R}^{d \times d}$ are symmetric matrices and $d$ is the total degree of $p$; in the homogeneous setting of hyperbolic polynomials this statement was known as the Lax conjecture, see [40]. We refer to [57] for a detailed survey and further references. The proof in [26] is based on the results of [56] and [7], see also [14], and uses algebro-geometrical techniques - the correspondence between (certain) determinantal representations of an irreducible plane curve and line bundles on its desingularization, together with a detailed analysis of the action of the complex conjugation on the Jacobian variety and the theory of Riemann's theta function; a new proof, using instead the theory of quadratic forms, has been discovered recently in [25. A somewhat weaker statement - namely, the existence of a representation (4.1) where now $A_{1}, A_{2} \in \mathbb{C}^{d \times d}$ are Hermitian matrices - has been established recently in [57, Section 5] and [48]; these proofs are also algebro-geometrical but avoid the transcendental machinery of Jacobian varieties and theta functions. In this section (Theorem 4.1), we provide a new proof (actually, two closely related proofs) of the existence of a representation (4.1) with $A_{1}, A_{2} \in \mathbb{C}^{d \times d}$ Hermitian matrices using factorizations of matrix valued polynomials. One advantage of our proof is that it provides a fairly constructive way to find such a representation. The most involved step is finding a stable factorization for a one-variable matrix polynomial that is positive semidefinite on the real line. As the latter can be implemented using any semidefinite programming package or a Riccati equation solver (see, e.g., [24] or [4. Section 2.7]), this construction can be easily implemented numerically, for instance in Matlab. For more on computational questions related to the construction of determinantal representations of real-zero polynomials, see [27, 46, 47, 41]. By a simple trick, Theorem 4.1. also implies the existence of a $2 d \times 2 d$ real symmetric representation for $p^{2}$ - see Remark 4.6.

Theorem 4.1. Let $p$ be a bivariate real-zero polynomial of total degree $d>0$ with $p(0,0)=1$. Then there exist $d \times d$ Hermitian matrices $A_{1}$ and $A_{2}$ so that (4.1) holds. 
We will need two lemmata. The first one is simply a restatement of one of the results of 45] in the non-homogeneous setting.

Lemma 4.2. Let $p$ be a real-zero polynomial of total degree $d$ and with $p(0,0)=1$. For every $\epsilon>0$ there exists a real-zero polynomial $q$ of total degree $d$ and with $q(0,0)=1$ such that each coefficient of $q$ is within $\epsilon$ distance of the corresponding coefficient of $p$, and for every $x_{2} \in \mathbb{R}$ the one-variable polynomial $\check{q}_{x_{2}}$ defined via

$$
\check{q}_{x_{2}}(t):=t^{d} q\left(1 / t, x_{2} / t\right)
$$

has only simple real zeros.

Proof. Let $\mathbf{p}\left(x_{0}, x_{1}, x_{2}\right):=x_{0}^{d} p\left(x_{1} / x_{0}, x_{2} / x_{0}\right)$. Then $\mathbf{p}$ is a degree $d$ homogeneous polynomial in three variables that is hyperbolic with respect to $e=(1,0,0)$, which means that $\mathbf{p}(e) \neq 0$ and for every $\left(x_{0}, x_{1}, x_{2}\right) \in \mathbb{R}^{3}$ the one-variable polynomial $t \rightarrow \mathbf{p}\left(x_{0}-t, x_{1}, x_{2}\right)$ has only real zeroes. By a result of [45], the polynomial $\mathbf{p}$ can be approximated arbitrarily close, in the sense of coefficients, by a degree $d$ homogeneous polynomial $\mathbf{q}$ which is strictly hyperbolic with respect to $e$; that is, $\mathbf{q}$ is hyperbolic with respect to $e$, and for every $\left(x_{0}, x_{1}, x_{2}\right) \in \mathbb{R}^{3}$ with $\left(x_{1}, x_{2}\right) \neq(0,0)$ the zeros of $t \rightarrow \mathbf{q}\left(x_{0}-t, x_{1}, x_{2}\right)$ are simple. But then $q\left(x_{1}, x_{2}\right):=$ $\mathbf{q}\left(1, x_{1}, x_{2}\right) / \mathbf{q}(1,0,0)$ has the desired property. Notice that while a priori the total degree of $q$ is at most $d$, it will be actually equal to $d$ if we choose $\epsilon$ small enough.

The following result is due to C. Hanselka [25]. For the sake of completeness, we include a proof.

Lemma 4.3. Let $M$ be a $d \times d$ matrix-valued polynomial in one variable with Hermitian coefficients, and assume that the polynomial $\operatorname{det}\left(t I_{d}-M(s)\right)$ has total degree at most $d$; then $M$ is linear (i.e., $\operatorname{deg} M \leq 1$ ).

Proof. Let

$$
\operatorname{det}\left(t I_{d}-M(s)\right)=t^{d}+p_{1}(s) t^{d-1}+\cdots+p_{d}(s),
$$

where $p_{j}$ is a polynomial of degree at most $j$. Assume that $M$ is a polynomial of degree $k$, and write $-M(s)=B_{0}+\cdots+B_{k} s^{k}$. The sum of $j \times j$ principal minors in $-M(s)$ is exactly $p_{j}(s)$; therefore the coefficient of $s^{k j}$ in $p_{j}(s)$ is the sum of $j \times j$ principal minors in $B_{k}$. But $\operatorname{deg} p_{j} \leq j$ for all $j$, hence if $k>1$ we conclude that the sum of $j \times j$ principal minors in $B_{k}$ is zero for all $j>0$. It follows that $B_{k}$ is nilpotent. Since $B_{k}$ is also Hermitian, it must be zero, a contradiction.

We will present two closely related proofs of Theorem 4.1 the first proof uses the Hermite matrix (considered in the context of real-zero polynomials and determinantal representations in [27] and in [43]), whereas the second proof uses intertwining polynomials and the Bezoutian (considered in this context in [57] and in [48, 38]).

First Proof of Theorem 4.1. We first claim that if we can establish the existence of a required determinantal representation for a dense subset of real-zero polynomials of total degree $d$ and with constant term 1 , then we are done 3 . Indeed, assume that we have real-zero polynomials $p^{(n)}, n \in \mathbb{N}$, of total degree $d$ with $p^{(n)}(0,0)=1$, so that the sequence $\left\{p^{(n)}\right\}_{n \in \mathbb{N}}$ converges

\footnotetext{
${ }^{3}$ This was previously noticed in [54, Lemma 8] and [48, Lemma 3.4]
} 
to $p$ and so that there exist Hermitian $d \times d$ matrices $A_{1}^{(n)}$ and $A_{2}^{(n)}$ with $p^{(n)}\left(x_{1}, x_{2}\right)=$ $\operatorname{det}\left(I_{d}+x_{1} A_{1}^{(n)}+x_{2} A_{2}^{(n)}\right)$. Let

$$
\mu:=\min \{|t|: p(t, 0) p(0, t)=0\} .
$$

Clearly, $\mu>0$. Then for $n$ large enough the spectra of $A_{1}^{(n)}$ and $A_{2}^{(n)}$ lie in the interval $\left(-2 \mu^{-1}, 2 \mu^{-1}\right)$. Since the spectral radius of an Hermitian matrix coincides with its operator $(2,2)$ norm, the matrices $A_{1}^{(n)}$ and $A_{2}^{(n)}$ have norms bounded by $2 \mu^{-1}$, and therefore the sequence $\left\{\left(A_{1}^{(n)}, A_{2}^{(n)}\right)\right\}_{n \in \mathbb{N}}$, has a limit point $\left(A_{1}, A_{2}\right)$. Then we get that $p\left(x_{1}, x_{2}\right)=\operatorname{det}\left(I_{d}+\right.$ $\left.x_{1} A_{1}+x_{2} A_{2}\right)$, with Hermitian $d \times d$ matrices $A_{1}$ and $A_{2}$, as desired.

Given $p$, we introduce

$$
\check{p}_{x_{2}}(t):=t^{d} p\left(1 / t, x_{2} / t\right)=t^{d}+p_{1}\left(x_{2}\right) t^{d-1}+\cdots+p_{d}\left(x_{2}\right) .
$$

One easily observes that $\operatorname{deg} p_{j} \leq j, j=1, \ldots, d$, and that for every $x_{2} \in \mathbb{R}$ the polynomial $\check{p}_{x_{2}}$ has only real zeros. Furthermore, we may assume by the previous paragraph and by Lemma 4.2 that for every $x_{2} \in \mathbb{R}$ the polynomial $\check{p}_{x_{2}}$ has only simple zeros.

Let $C\left(x_{2}\right)$ be the companion matrix

$$
C\left(x_{2}\right)=\left[\begin{array}{cccc}
0 & \cdots & 0 & -p_{d}\left(x_{2}\right) \\
1 & & 0 & -p_{d-1}\left(x_{2}\right) \\
& \ddots & & \vdots \\
0 & & 1 & -p_{1}\left(x_{2}\right)
\end{array}\right] .
$$

Then

$$
\check{p}_{x_{2}}(t)=\operatorname{det}\left(t I_{d}-C\left(x_{2}\right)\right)
$$

Denote the zeros of $\check{p}_{x_{2}}$ by $\lambda_{1}\left(x_{2}\right), \ldots, \lambda_{d}\left(x_{2}\right)$, and let $s_{j}\left(x_{2}\right)$ be their $j$ th Newton sum:

$$
s_{j}\left(x_{2}\right)=\sum_{k=1}^{d} \lambda_{k}\left(x_{2}\right)^{j}, \quad j=0,1, \ldots
$$

As is well known, $s_{j}\left(x_{2}\right)$ can be expressed in terms of $p_{j}\left(x_{2}\right)$, as follows

$$
s_{0}\left(x_{2}\right)=d, \quad s_{1}\left(x_{2}\right)=-p_{1}(z), \quad s_{j}\left(x_{2}\right)=-j p_{j}\left(x_{2}\right)-\sum_{k=1}^{j-1} p_{k}\left(x_{2}\right) s_{j-k}\left(x_{2}\right), \quad j=2, \ldots, d .
$$

Note that $s_{j}$ is a polynomial of degree $\leq j, j=0, \ldots, d$. We let $H\left(x_{2}\right)$ be the Hermite matrix of $\check{p}_{x_{2}}$, namely (see, e.g., [34]) the Hankel matrix whose entries are the Newton sums of the zeros of $\check{p}_{x_{2}}$ :

$$
H\left(x_{2}\right)=\left[s_{i+j}\left(x_{2}\right)\right]_{i, j=0, \ldots, d-1} .
$$

Clearly, $H$ is a matrix polynomial of degree at most $2 d$. E.g., for $d=2$ we have

$$
H\left(x_{2}\right)=\left[\begin{array}{cc}
2 & -p_{1}\left(x_{2}\right) \\
-p_{1}\left(x_{2}\right) & p_{1}\left(x_{2}\right)^{2}-2 p_{2}\left(x_{2}\right)
\end{array}\right] \text {. }
$$

Since all the zeros of $\check{p}_{x_{2}}$ are real and simple for real $x_{2}$, we have that $H\left(x_{2}\right)>0, x_{2} \in \mathbb{R}$. This is well known and it follows immediately from

$$
H\left(x_{2}\right)=V\left(x_{2}\right)^{T} V\left(x_{2}\right),
$$


where $V\left(x_{2}\right)$ is the (real) Vandermonde matrix

$$
V\left(x_{2}\right)=\left[\lambda_{k+1}\left(x_{2}\right)^{j}\right]_{k, j=0, \ldots, d-1} .
$$

In addition, one may easily check (e.g., using (4.2)) that

$$
C\left(x_{2}\right)^{T} H\left(x_{2}\right)=H\left(x_{2}\right) C\left(x_{2}\right) .
$$

By the positive definiteness of $H\left(x_{2}\right)$ for all real $x_{2}$, we may factor $H\left(x_{2}\right)$ as

$$
H\left(x_{2}\right)=Q\left(x_{2}\right)^{*} Q\left(x_{2}\right), \quad x_{2} \in \mathbb{R},
$$

where $Q\left(x_{2}\right)$ is a matrix polynomial of degree $d$ and $Q\left(x_{2}\right)$ is invertible for $\operatorname{Im} x_{2} \geq 0$; see, for instance, [52]. We now let

$$
M\left(x_{2}\right)=Q\left(x_{2}\right) C\left(x_{2}\right) Q\left(x_{2}\right)^{-1},
$$

and obtain that

$$
\check{p}_{x_{2}}(t)=\operatorname{det}\left(t I_{d}-M\left(x_{2}\right)\right) .
$$

Note that $M\left(x_{2}\right)=M\left(x_{2}\right)^{*}$ for $x_{2} \in \mathbb{R}$. Indeed, (4.4) implies that

$$
C\left(x_{2}\right)^{*} Q\left(x_{2}\right)^{*} Q\left(x_{2}\right)=Q\left(x_{2}\right)^{*} Q\left(x_{2}\right) C\left(x_{2}\right), \quad x_{2} \in \mathbb{R} .
$$

Multiplying on the left with $Q\left(x_{2}\right)^{*-1}$ and on the right with $Q\left(x_{2}\right)^{-1}$, yields that $M\left(x_{2}\right)=$ $M\left(x_{2}\right)^{*}, x_{2} \in \mathbb{R}$.

Next, we claim that the rational matrix function $M\left(x_{2}\right)$ is in fact a matrix polynomial. The only possible poles arise from the zeros of $Q\left(x_{2}\right)$. Let $a$ be a zero of $Q\left(x_{2}\right)$. Then $\operatorname{Im} a<0$. We rewrite (4.5) as

$$
H\left(x_{2}\right)=Q\left(\bar{x}_{2}\right)^{*} Q\left(x_{2}\right)
$$

for all $x_{2} \in \mathbb{C}$, and substitute in (4.4), obtaining

$$
C\left(x_{2}\right)^{T} Q\left(\bar{x}_{2}\right)^{*}=Q\left(\bar{x}_{2}\right)^{*} Q\left(x_{2}\right) C\left(x_{2}\right) Q\left(x_{2}\right)^{-1}=Q\left(\bar{x}_{2}\right)^{*} M\left(x_{2}\right),
$$

for all $x_{2} \in \mathbb{C}$. Since $Q(\bar{a})$ is invertible, we conclude that

$$
M\left(x_{2}\right)=Q\left(\bar{x}_{2}\right)^{*-1} C\left(x_{2}\right)^{T} Q\left(\bar{x}_{2}\right)^{*}
$$

is regular at $a$, i.e., $a$ is not a pole of $M\left(x_{2}\right)$.

It follows now from Lemma 4.3 that $\operatorname{deg} M \leq 1$, i.e., we can write

$$
M\left(x_{2}\right)=-A_{1}-A_{2} x_{2},
$$

where $A_{1}$ and $A_{2}$ are $d \times d$ Hermitian matrices. Then

$$
\check{p}_{x_{2}}(t)=\operatorname{det}\left(t I_{d}+A_{1}+A_{2} x_{2}\right),
$$

and thus

$$
p\left(x_{1}, x_{2}\right)=x_{1}^{d} \check{p}_{\frac{x_{2}}{x_{1}}}\left(\frac{1}{x_{1}}\right)=\operatorname{det}\left(I_{d}+x_{1} A_{1}+x_{2} A_{2}\right)
$$

Note that the proof provides a constructive way to find a representation (4.1). We illustrate this with an example. 
Example 4.4. Let $p(x, y)=1+10 y+4 x-y^{2}-2 x y-x^{2}$. Then $\check{p}_{y}(t)=t^{2}+(10 y+4) t+$ $\left(-1-2 y-y^{2}\right)$. We get

$$
H(y)=\left[\begin{array}{cc}
2 & -10 y-4 \\
-10 y-4 & 102 y^{2}+84 y+18
\end{array}\right]
$$

Factoring as in (4.5) we find that

$$
Q(y)=\left[\begin{array}{cc}
\sqrt{2} & -2 \sqrt{2}-5 \sqrt{2} y \\
0 & \sqrt{10}\left(1+y \frac{11+3 i}{5}\right)
\end{array}\right]
$$

Then

$$
M(y)=Q(y) C(y) Q(y)^{-1}=\left[\begin{array}{cc}
-5 y-2 & \sqrt{5}\left(1+\frac{y}{5}(11-3 i)\right) \\
\sqrt{5}\left(1+\frac{y}{5}(11+3 i)\right) & -5 y-2
\end{array}\right] .
$$

Ultimately, we find that

$$
p(x, y)=\operatorname{det}\left(I_{2}+x\left[\begin{array}{cc}
2 & -\sqrt{5} \\
-\sqrt{5} & 2
\end{array}\right]+y\left[\begin{array}{cc}
5 & \frac{\sqrt{5}}{5}(-11+3 i) \\
\frac{\sqrt{5}}{5}(-11-3 i) & 5
\end{array}\right]\right) .
$$

By the way, the polynomial $p$ was constructed using $A_{1}=\left[\begin{array}{ll}1 & 2 \\ 2 & 3\end{array}\right]$ and $A_{2}=\left[\begin{array}{ll}4 & 5 \\ 5 & 6\end{array}\right]$.

Before presenting a second proof of Theorem 4.1, we introduce a definition. Let $p$ be a real-zero polynomial of total degree $d, p(0,0)=1$, and let $q$ be a real-zero polynomial of total degree less than $d, q(0,0)>0$. We define

$$
\check{p}_{x_{2}}(t):=t^{d} p\left(1 / t, x_{2} / t\right), \quad \check{q}_{x_{2}}(t):=t^{d-1} q\left(1 / t, x_{2} / t\right),
$$

and let, for $x_{2} \in \mathbb{R}, \lambda_{1}\left(x_{2}\right) \leq \cdots \leq \lambda_{d}\left(x_{2}\right)$ and $\mu_{1}\left(x_{2}\right) \leq \cdots \leq \mu_{d-1}\left(x_{2}\right)$ be the zeros of $\check{p}_{x_{2}}$ and of $\check{q}_{x_{2}}$, respectively, counting multiplicities. We will say that $q$ interlaces $p$ if

$$
\lambda_{1}\left(x_{2}\right) \leq \mu_{1}\left(x_{2}\right) \leq \lambda_{2}\left(x_{2}\right) \leq \cdots \leq \lambda_{d-1}\left(x_{2}\right) \leq \mu_{d-1}\left(x_{2}\right) \leq \lambda_{d}\left(x_{2}\right)
$$

for all $x_{2} \in \mathbb{R}$. We will say that $q$ strictly interlaces $p$ if all the zeros of $\check{p}_{x_{2}}$ are simple and strict inequalities hold in (4.7), for all $x_{2} \in \mathbb{R}$.

As an example, let $p$ be a real-zero polynomial of total degree $d, p(0,0)=1$, and let $\left(x_{1}^{0}, x_{2}^{0}\right)$ belong to the connected component of $(0,0)$ in $\left\{\left(x_{1}, x_{2}\right) \in \mathbb{R}^{2}: p\left(x_{1}, x_{2}\right)>0\right\}$. We set $\mathbf{p}\left(x_{0}, x_{1}, x_{2}\right)=x_{0}^{d} p\left(x_{1} / x_{0}, x_{2} / x_{0}\right)$ and define

$$
q\left(x_{1}, x_{2}\right)=\left.\frac{d}{d s} \mathbf{p}\left(1+s, x_{1}+s x_{1}^{0}, x_{2}+s x_{2}^{0}\right)\right|_{s=0} ;
$$

$q$ is called the Renegar derivative of $p$ with respect to $\left(x_{1}^{0}, x_{2}^{0}\right)$ and it interlaces $p$; see [50, 42]. The interlacing is strict if all the zeros of $\check{p}_{x_{2}}$ are simple for all $x_{2} \in \mathbb{R}$. Notice that for $\left(x_{1}^{0}, x_{2}^{0}\right)=(0,0)$, we have simply $\check{q}_{x_{2}}=\left(\check{p}_{x_{2}}\right)^{\prime}$.

Second Proof of Theorem 4.1. We assume as in the first proof that $p$ is a real-zero polynomial of total degree $d, p(0,0)=1$, such that the polynomial $\check{p}_{x_{2}}$ has only simple zeros for all $x_{2} \in \mathbb{R}$. We choose a real-zero polynomial $q$ of total degree less than $d, q(0,0) \neq 0$, that strictly interlaces $p$. We let $B\left(x_{2}\right)$ be the Bezoutian of the polynomials $\check{q}_{x_{2}}$ and $\check{p}_{x_{2}}$, namely (see, e.g., [34])

$$
B\left(x_{2}\right)=\left[b_{i j}\left(x_{2}\right)\right]_{i, j=0, \ldots, d-1},
$$


where $b_{i j}\left(x_{2}\right)$ are determined from

$$
\frac{\check{q}_{x_{2}}(t) \check{p}_{x_{2}}(s)-\check{q}_{x_{2}}(s) \check{p}_{x_{2}}(t)}{t-s}=\sum_{i, j=0, \ldots, d-1} b_{i j}\left(x_{2}\right) t^{i} s^{j} .
$$

It is easily seen that $b_{i j}\left(x_{2}\right)$ are polynomials (over $\mathbb{Z}$ ) in the coefficients of $\check{p}_{x_{2}}$ and $\check{q}_{x_{2}}$, hence polynomials in $x_{2}$, i.e., $B$ is a matrix polynomial. The defining equation (4.8) can be conveniently rewritten as

$$
v_{d-1}(t) B\left(x_{2}\right) v_{d-1}(s)^{T}=\frac{\check{q}_{x_{2}}(t) \check{p}_{x_{2}}(s)-\check{q}_{x_{2}}(s) \check{p}_{x_{2}}(t)}{t-s}
$$

and taking the limit $t \rightarrow s$,

$$
v_{d-1}(s) B\left(x_{2}\right) v_{d-1}(s)^{T}=\left(\check{q}_{x_{2}}\right)^{\prime}(s) \check{p}_{x_{2}}(s)-\check{q}_{x_{2}}(s)\left(\check{p}_{x_{2}}\right)^{\prime}(s),
$$

where

$$
v_{d-1}(t)=\left[\begin{array}{llll}
1 & t & \cdots & t^{d-1}
\end{array}\right]
$$

Since the zeros of $\check{p}_{x_{2}}$ and $\check{q}_{x_{2}}$ are real, simple, and alternate for real $x_{2}$, we have that $B\left(x_{2}\right)>0, x_{2} \in \mathbb{R}$. This well known fact can be seen immediately by using (4.9)-(4.10) to compute

$$
V\left(x_{2}\right) B\left(x_{2}\right) V\left(x_{2}\right)^{T}=\operatorname{diag}\left(-\check{q}_{x_{2}}\left(\lambda_{i}\left(x_{2}\right)\right)\left(\check{p}_{x_{2}}\right)^{\prime}\left(\lambda_{i}\left(x_{2}\right)\right)\right)_{i=1, \ldots, d},
$$

where $V\left(x_{2}\right)$ is the Vandermonde matrix (4.3) based at the zeros of $\check{p}_{x_{2}}$. In addition, one may easily check

$$
C\left(x_{2}\right) B\left(x_{2}\right)=B\left(x_{2}\right) C\left(x_{2}\right)^{T}
$$

- e.g., multiplying both sides by $V\left(x_{2}\right)$ from the left and $V\left(x_{2}\right)^{T}$ from the right, and using (4.9) $-(4.10)$.

By the positive definiteness of $B\left(x_{2}\right)$ for all real $x_{2}$, we may factor $B\left(x_{2}\right)$ as

$$
B\left(x_{2}\right)=P\left(x_{2}\right) P\left(x_{2}\right)^{*}, \quad x_{2} \in \mathbb{R},
$$

where $P\left(x_{2}\right)$ is a matrix polynomial and $P\left(x_{2}\right)$ is invertible for $\operatorname{Im} x_{2} \geq 0$, and we let

$$
M\left(x_{2}\right)=P\left(x_{2}\right)^{-1} C\left(x_{2}\right) P\left(x_{2}\right),
$$

and obtain that

$$
\check{p}_{x_{2}}(t)=\operatorname{det}\left(t I_{d}-M\left(x_{2}\right)\right) .
$$

As in the first proof of the theorem, (4.11) and (4.12) imply that $M\left(x_{2}\right)=M\left(x_{2}\right)^{*}, x_{2} \in \mathbb{R}$, and that the rational matrix function $M\left(x_{2}\right)$ is regular at a zero $a$ of $P\left(x_{2}\right)$, so that it is in fact a matrix polynomial 4 . It follows from Lemma 4.3 that $M$ is linear:

$$
M\left(x_{2}\right)=-A_{1}-A_{2} x_{2},
$$

where $A_{1}$ and $A_{2}$ are $d \times d$ Hermitian matrices, and then

$$
p\left(x_{1}, x_{2}\right)=x_{1}^{d} \check{p}_{\frac{x_{2}}{x_{1}}}\left(\frac{1}{x_{1}}\right)=\operatorname{det}\left(I_{d}+x_{1} A_{1}+x_{2} A_{2}\right) .
$$

\footnotetext{
${ }^{4}$ Alternatively, we can prove that a zero $a$ of $P\left(x_{2}\right)$ is not a pole of $M\left(x_{2}\right)$ similarly to the proof of the claim in the proof of Theorem 2.1. It is well known that $\operatorname{det} B(a)=0$ iff the polynomials $\check{p}_{a}$ and $\check{q}_{a}$ have a common zero $\lambda$; let us assume that $\lambda$ is a simple zero of both $\check{p}_{a}$ and $\check{q}_{a}$, then it is also well known that the left kernel of $B(a)$ is spanned by $v_{d-1}(\lambda)$ (all these facts follow quite easily from (4.9)-(4.10)). Since $B(a)=P(a) P(\bar{a})^{*}$, and since $v_{d-1}(\lambda) C(a)=\lambda v_{d-1}(\lambda)$, it follows that the one-dimensional left kernel of $P(a)$ is the left eigenspace of $C(a)$, implying as in the proof of Theorem 2.1 that $a$ is not a pole of $M\left(x_{2}\right)$.
} 
This second proof of Theorem 4.1 is of course constructive as well as soon as we choose a strictly interlacing polynomial $q$.

We notice also that the algebro-geometrical proof of Theorem 4.1 given in [57] and in [48] also uses an interlacing polynomial $q$, and yields a determinantal representation with

$$
q\left(x_{1}, x_{2}\right)=c \operatorname{adj}\left(I+x_{1} A_{1}+x_{2} A_{2}\right) c^{T},
$$

where adj denotes the classical adjoint or adjugate matrix (the matrix of cofactors) and $c \in \mathbb{C}^{1 \times d}$. It would be interesting to see whether this relation holds for the determinantal representation constructed in the second proof of Theorem 4.1 above (meaning that the two constructions are essentially equivalent, despite using quite different methods).

Remark 4.5. Note that for $d=2$ we can always convert a representation $p\left(x_{1}, x_{2}\right)=\operatorname{det}\left(I_{2}+\right.$ $x_{1} A_{1}+x_{2} A_{2}$ ) with $A_{1}$ and $A_{2}$ Hermitian, to one with real symmetric $A_{1}$ and $A_{2}$. Indeed, write $A_{1}=U D U^{*}$, with $U$ unitary and $D$ diagonal, and consider $U^{*} A_{2} U$ which has a complex $(1,2)$ entry with, say, argument $\theta$. Then letting $V=\left[\begin{array}{cc}1 & 0 \\ 0 & e^{i \theta}\end{array}\right]$ and $\hat{A}_{1}=D=V D V^{*}, \hat{A}_{2}=$ $V U A_{2} U^{*} V^{*} \in \mathbb{R}^{2 \times 2}$, we obtain $p\left(x_{1}, x_{2}\right)=\operatorname{det}\left(I_{2}+x \hat{A}_{1}+x_{2} \hat{A}_{2}\right)$, as desired.

Remark 4.6. (See [49, Section 1.4] and [44, Lemma 2.14].) From the representation as in Theorem 4.1, we may represent $p\left(x_{1}, x_{2}\right)^{2}$ as

$$
p\left(x_{1}, x_{2}\right)^{2}=\operatorname{det}\left(I_{2 d}+x_{1} \alpha_{1}+x_{2} \alpha_{2}\right),
$$

where $\alpha_{1}=\alpha_{1}^{T}, \alpha_{2}=\alpha_{2}^{T} \in \mathbb{R}^{2 d \times 2 d}$. Indeed, with $A_{1}$ and $A_{2}$ as in Theorem 4.1, we write

$$
A_{1}=A_{1 R}+i A_{1 I}, A_{2}=A_{2 R}+i A_{2 I},
$$

where $A_{1 R}, A_{2 R}, A_{1 I}, A_{2 I} \in \mathbb{R}^{d \times d}$. It is easy to check that since $A_{1}$ and $A_{2}$ are Hermitian, $A_{1 R}, A_{2 R}$ are symmetric and $A_{1 I}, A_{2 I}$ are skew-symmetric. Let now

$$
\alpha_{1}=\left[\begin{array}{cc}
A_{1 R} & A_{1 I} \\
-A_{1 I} & A_{1 R}
\end{array}\right], \quad \alpha_{2}=\left[\begin{array}{cc}
A_{2 R} & A_{2 I} \\
-A_{2 I} & A_{2 R}
\end{array}\right]
$$

and (4.13) follows. Indeed, using

$$
U=\frac{1}{\sqrt{2}}\left[\begin{array}{cc}
I_{d} & I_{d} \\
i I_{d} & -i I_{d}
\end{array}\right]
$$

it is easy to check that

$$
U\left[\begin{array}{cc}
A_{1} & 0 \\
0 & A_{1}^{T}
\end{array}\right] U^{*}=\alpha_{1}, \quad U\left[\begin{array}{cc}
A_{2} & 0 \\
0 & A_{2}^{T}
\end{array}\right] U^{*}=\alpha_{2} .
$$

\section{REFERENCES}

[1] J. Agler, On the representation of certain holomorphic functions defined on a polydisc, In Topics in operator theory: Ernst D. Hellinger Memorial Volume, Oper. Theory Adv. Appl., Vol. 48, pp. 47-66, Birkhäuser, Basel, 1990.

[2] J. Agler and J. E. McCarthy, Distinguished varieties, Acta Math. 194 (2005), no. 2, 133-153.

[3] D. Z. Arov. Passive linear steady-state dynamical systems. (Russian) Sibirsk. Mat. Zh. 20 (1979), no. $2,211-228,457$.

[4] M. Bakonyi and H. J. Woerdeman. Matrix completions, moments, and sums of Hermitian squares. Princeton University Press, Princeton, NJ, 2011. 
[5] J. A. Ball, I. Gohberg, and L. Rodman. Interpolation of rational matrix functions. Operator Theory: Advances and Applications, 45. Birkhäuser Verlag, Basel, 1990. xii+605 pp.

[6] J. A. Ball, C. Sadosky, and V. Vinnikov. Scattering systems with several evolutions and multidimensional input/state/output systems. Integral Equations Operator Theory 52:323-393, no. 3, 2005.

[7] J. A. Ball and V. Vinnikov. Zero-pole interpolation for meromorphic matrix functions on a compact Riemann surface and a matrix Fay trisecant identity. Amer. J. Math. 121:841-888, 1999.

[8] H. Bart, I. Gohberg, and M. A. Kaashoek. Minimal factorization of matrix and operator functions. Operator Theory: Adv. Appl., 1. Birkhäuser Verlag, Basel-Boston, Mass., 1979

[9] S. Basu and A. Fettweis. New results on stable multidimensional polynomials. II. Discrete case. IEEE Trans. Circuits and Systems 34:1264-1274, 1987.

[10] K. Bickel and G. Knese. Fundamental Agler decompositions. Integral Equations Oper. Theory, to appear.

[11] J. Borcea, P. Brändén, and T. M. Liggett. Negative dependence and the geometry of polynomials. $J$. Amer. Math. Soc. 22:521-567, no. 2, 2009.

[12] J. C. Doyle. Analysis of feedback systems with structured uncertainties. Proc. IEE-D 129:242-250, no. $6,1982$.

[13] M. A. Dritschel and J. Rovnyak, The operator Fejér-Riesz theorem, Operator Theory: Adv. Appl. 207 (2010), pp 223-254.

[14] B. A. Dubrovin. Matrix finite zone operators. Contemporary Problems of Mathematics (Itogi Nauki i Techniki) 23, pp. 33-78 (1983) (Russian).

[15] W. Fulton. Algebraic Curves. Mathematics Lecture Note Series. W.A. Benjamin, 1974.

[16] L. Gårding. Linear hyperbolic partial differential equations with constant coefficients. Acta Math. 85:262, 1951.

[17] L. Gårding. An inequality for hyperbolic polynomials. J. Math. Mech. 8:957-965, 1959.

[18] J. S. Geronimo, P. Iliev, and G. Knese. Polynomials with no zeros on a face of the bidisk. arXiv 1301.3510 .

[19] J. S. Geronimo and H. J. Woerdeman. Positive extensions, Fejér-Riesz factorization and autoregressive filters in two variables. Ann. of Math. (2), 160(3):839-906, 2004.

[20] J. S. Geronimo and H. J. Woerdeman. Two-variable polynomials: intersecting zeros and stability. IEEE Trans. Circuits Syst. 53(5):1130-1139, 2006.

[21] D. D. Givone and R. P. Roesser, Multidimensional linear iterative circuits-general properties, IEEE Trans. Computers 21: 1067-1073, 1972.

[22] A. Grinshpan, D. S. Kaliuzhnyi-Verbovetskyi, and H. J. Woerdeman. Norm-constrained determinantal representations of multivariable polynomials. Complex Anal. Oper. Theory 7 (2013), 635-654.

[23] L. Gurvits. Van der Waerden/Schrijver-Valiant like conjectures and stable (aka hyperbolic) homogeneous polynomials: one theorem for all. With a corrigendum. Electron. J. Combin., 15 (2008), no. 1, Research Paper 66, 26 pp.

[24] Y. Hachez and H. J. Woerdeman. The Fischer-Frobenius transformation and outer factorization. Operator theory, structured matrices, and dilations, 181-203, Theta Ser. Adv. Math., 7, Theta, Bucharest, 2007.

[25] C. Hanselka. Ph. D. Thesis, University of Konstanz (in preparation).

[26] J. W. Helton and V. Vinnikov. Linear matrix inequality representation of sets. Comm. Pure Appl. Math. 60 (2007), 654-674.

[27] D. Henrion. Detecting rigid convexity of bivariate polynomials. Linear Algebra Appl. 432:1218-1233, 2010.

[28] A. Horn, On the eigenvalues of a matrix with prescribed singular values. Proc. Amer. Math. Soc. 5, (1954). $4-7$.

[29] R. A. Horn and C. R. Johnson, Topics in matrix analysis. Cambridge University Press, Cambridge, 1991. viii+607 pp.

[30] T. Kailath, A. Vieira, and M. Morf, Inverses of Toeplitz operators, innovations, and orthogonal polynomials, SIAM Rev. 20 (1978), 106-119.

[31] R. E. Kalman, P. L. Falb, and M. A. Arbib. Topics in mathematical system theory. McGraw-Hill Book Co., New York-Toronto, Ont.-London 1969 xiv+358 pp. 
[32] D. Kerner and V. Vinnikov. Determinantal representations of singular hypersurfaces in $\mathbb{P}^{n}$. Adv. Math. 231:1619-1654, 2012.

[33] G. Knese. Bernstein-Szegö measures on the two-dimensional torus. Indiana Univ. Math. J. 57(3):1353$1376,2008$.

[34] M. G. Krein and M. A. Naimark. The method of symmetric and Hermitian forms in the theory of the separation of the roots of algebraic equations. Kharkov, 1936. English translation (by O. Boshko and J. L. Howland): Lin. Mult. Alg. 10:265-308, 1981.

[35] A. Kummert. A parametric representation for $k$-variable Schur polynomials. IEEE Trans. Circuits Systems 37:1288-1291, no. 10, 1990.

[36] A. Kummert. Synthesis of two-dimmensional lossless $m$-ports with prescribed scattering matrix. Circuits Systems Signal Processing 8:97-119, no. 1, 1989.

[37] A. Kummert. 2-D stable polynomials with parameter-dependent coefficients: generalizations and new results. IEEE Trans. Circuits Systems I: Fund. Theory Appl. 49:725-731, 2002.

[38] M. Kummer, D. Plaumann, and C. Vinzant. Hyperbolic polynomials, interlacers, and sums of squares. arXiv:1212.6696.

[39] P. Lancaster and M. Tismenetsky. The theory of matrices. Second edition. Computer Science and Applied Mathematics. Academic Press, Inc., Orlando, FL, 1985.

[40] A. S. Lewis, P. A. Parrilo, and M. V. Ramana. The Lax conjecture is true. Proc. Amer. Math. Soc. 133 (2005), 2495-2499.

[41] A. Leykin and D. Plaumann. Determinantal representations of hyperbolic curves via polynomial homotopy continuation. arXiv:1212.3506.

[42] T. Netzer, D. Plaumann, and M. Schweighofer. Exposed faces of semidefinitely representable sets. SIAM J. Optim. 20:1944-1955, 2010.

[43] T. Netzer, D. Plaumann and A. Thom. Determinantal representations and the Hermite matrix. Michigan Math. J. 62 (2013), 407-420

[44] T. Netzer, A. Thom: Polynomials with and without determinantal representations. Linear Algebra Appl. 437 (2012), 1579-1595.

[45] W. Nuij. A note on hyperbolic polynomials. Math. Scand. 23 (1968), 69-72.

[46] D. Plaumann, B. Sturmfels, and C. Vinzant. Quartic Curves and Their Bitangents. J. Symb. Comp. 46 (2011), 712-733.

[47] D. Plaumann, B. Sturmfels, and C. Vinzant. Computing Linear Matrix Representations of HeltonVinnikov Curves. Operator Theory: Adv. Appl. 222 (Festschrift in honor of J. William Helton), 259-277 (2012).

[48] D. Plaumann and C. Vinzant. Determinantal representations of hyperbolic plane curves: An elementary approach. arXiv:1207.7047.

[49] M. Ramana and A. J. Goldman. Some geometric results in semidefinite programming. J. Global Optim. 7:33-50, 1995.

[50] J. Renegar. Hyperbolic programs, and their derivative relaxations. Found. Comput. Math. 6:59-79, 2006.

[51] M. Rosenblatt, A multi-dimensional prediction problem, Ark. Mat. 3 (1958), 407-424.

[52] M. Rosenblum and J. Rovnyak, Hardy Classes and Operator Theory. Oxford Mathematical Monographs. Oxford Science Publications. The Clarendon Press, Oxford University Press, New York, 1985.

[53] W. Rudin. Function Theory on Polydiscs. W. A. Benjamin, Inc., New York-Amsterdam, 1969.

[54] D. Speyer. Horns problem, Vinnikov curves, and the hive cone. Duke Math. J. 127(3) (2005), 395-427.

[55] V. Vinnikov. Complete description of determinantal representations of smooth irreducible curves. Linear Algebra Appl. 125:103-140, 1989.

[56] V. Vinnikov. Self-adjoint determinantal representions of real plane curves. Math. Ann. 296:453-479, 1993.

[57] V. Vinnikov. LMI representations of convex semialgebraic sets and determinantal representations of algebraic hypersurfaces: past, present, and future, Operator Theory: Adv. Appl. 222 (Festschrift in honor of J. William Helton), 325-349 (2012).

[58] D. G. Wagner. Multivariate stable polynomials: theory and applications. Bull. Amer. Math. Soc. 48:5384, no. 1, 2011. 
[59] H. J. Woerdeman. Determinantal representations of stable polynomials. Operator Theory: Adv. Appl. 237 (2013), 241-246.

Department of Mathematics, Drexel University, 3141 Chestnut St., Philadelphia, PA, 19104

E-mail address: \{tolya,dmitryk, hugo\}@math.drexel.edu

Department of Mathematics, Ben-Gurion University of the Negev, Beer-Sheva, Israel, 84105

E-mail address: vinnikov@math.bgu.ac.il 